\title{
Electrically injected quantum dot photonic crystal microcavity light emitters and microcavity arrays
}

\author{
S Chakravarty, P Bhattacharya, J Topol'ančik and Z Wu \\ Solid State Electronics Laboratory, Department of Electrical Engineering and \\ Computer Science, University of Michigan, Ann Arbor, MI 48109-2122, USA \\ E-mail:pkb@eecs.umich.edu
}

Received 10 August 2006, in final form 2 October 2006

Published 19 April 2007

Online at stacks.iop.org/JPhysD/40/2683

\begin{abstract}
Progress in the design, fabrication and characterization of electrically injected photonic-crystal quantum-dot microcavity light sources is described. In the devices investigated in this study, the carriers are injected directly into the photonic crystal microcavity, avoiding the surface state recombination in the photonic crystal pattern. A novel and robust air-bridge contact technology is demonstrated. Spectral linewidths $\sim 2-3 \mathrm{~nm}$ are observed from hexagonal microcavities of varying sizes in the output spectra of oxide-clad microcavity devices. Narrower linewidths $\sim 1.3 \mathrm{~nm}$ are observed from air-clad devices. Results on arrays of densely packed oxide-clad photonic crystal microcavities are also presented. Spectral characteristics of oxide-clad and air-clad devices are compared.
\end{abstract}

(Some figures in this article are in colour only in the electronic version)

\section{Introduction and background}

Photonic crystals (PCs) are gradually emerging as feasible solutions in a wide range of applications including single photon sources [1], lasers [2], filters [3], low-loss waveguides [4], sensors [5,6] and optical interconnects [7]. PC devices have been the subject of intense research and development for nearly a decade, as an effective medium for studying lightmatter interactions in the solid state $[8,9]$. By engineering point or line defects in periodic photonic lattices, localized modes are created in a photonic band gap (PBG) that allow one to confine and manipulate the propagation of light on length scales of the order of the wavelength of light. Precise control of frequencies at which electromagnetic radiation is either enhanced [10] or inhibited [11] can be achieved by tuning the geometry of the periodic lattice or what is also known as PBG defect engineering. Advances in nano-fabrication have made possible the construction of PCs with bandgaps in the optical regime of the electromagnetic spectrum. While reliable fabrication of three-dimensional (3D) PC microcavities is a challenge with current lithography techniques $[12,13]$, many desirable properties of optical elements have been realized in two-dimensional (2D) PC slab microcavities [1-7] where horizontal confinement is provided by the 2D PC and vertical confinement is achieved by total internal reflection.

The most significant application of a $2 \mathrm{D} \mathrm{PC}$ is a microcavity light source (laser/ light-emitting diode) with selforganized quantum dot (QD) as the light emitting material. QDs, unlike quantum wells (QWs), provide 3D confinement of carriers, thereby leading to narrow emission linewidths with nearly singular density of states. Combining the technology of self-organized QDs with PBG defect mode engineering leads to the prospect of fabricating advanced light emitters with single mode operation, higher efficiency, lower thresholds, higher bandwidths and increased output directionality. PC nano-emitters with a single embedded QD hold tremendous prospects for studying fundamental light-matter interactions in semiconductor systems as also in quantum computation [14], quantum cryptography [15] and quantum teleportation [16].

Optically pumped single-mode PC microcavity lasers were demonstrated at $1.55 \mu \mathrm{m}$ for the first time by Painter et al using compressively strained InGaAsP QWs as the active material [2]. QDs were later incorporated successfully in PC microcavities to demonstrate lasing from single [17] and coupled [18] PC cavities. Vertical optical confinement in optically excited devices is provided by uniform air or oxide 
cladding above and below the active region. In electrically injected PC devices, the fabrication becomes more challenging due to the need for a conducting aperture in the air or oxide cladding below the microcavity to provide a path for carrier transport. Resonant modes in the microcavity with antinodes at the cavity centre are suppressed due to poor vertical confinement by the high index post in the PBG. Modes with an electric field profile that has a node at the centre, and the maximum of the electric field energy density around the periphery of the microcavity have the necessary vertical optical confinement and are consequently enhanced. Also, depending on the material used and the doping, the conducting aperture (post) can increase the resistance to the flow of carriers. A reliable electrical carrier injection scheme is necessary to excite suitable modes for enhanced emission.

The first electrically injected surface-emitting 2D PC microcavity light source with multiquantum well emission at $0.94 \mu \mathrm{m}$ was demonstrated by Zhou et al [19]. Thereafter, a 2D PC microcavity light emitter with QD active region was demonstrated by Sabarinathan et al [20]. Major challenges in the design and fabrication of those devices included the complex heterostructure, difficult 3D defect mode calculations requiring large computational domains and significantly larger etch depths required for sufficient vertical mode confinement than those required in free-standing PC slabs. However, the advantages were good mechanical and thermal stability.

An electrically injected single-mode microcavity laser with a QW active region [21] was later demonstrated by employing a doped dielectric micropillar under a free-standing PC slab microcavity. In all these electrically injected microcavity light emitters that have been reported, large carrier loss occurs in the surface states as the injected carriers traverse the etched surfaces of the photonic crystal to reach the active region [19-21], thereby reducing the carrier injection efficiency. The surface scattering related loss was also a carrier loss component in the first demonstrated electrically injected QW single cell PC microcavity laser [21]. We describe here the characteristics of electrically injected PC microcavity light sources in which the carrier injection related loss is reduced. In the first device, a bottom-emitting configuration was considered where the p-metal contact pad was placed directly above the microcavity and the emitted light was collected from the polished rear surface of the wafer [22]. In the second device, light emission from the top surface was achieved with an air-bridge assisted metal contact. The robust air-bridge connects the p-contact on the microcavity to a contact pad outside the air-holes of the PC. Coherent emission is observed under certain biasing conditions, corresponding to the resonant modes of the microcavity. Electrically injected arrays of PC microcavity light sources, connected by the airbridges are also demonstrated for the first time. Both electrical carrier injection schemes avoid carrier scattering losses at the etched surface of the PC.

\section{Bottom-emitting QD PC microcavity light emitter}

\subsection{Design and fabrication}

The bottom emitting device, shown schematically in figure 1(a), is fabricated with a GaAs-based heterostructure

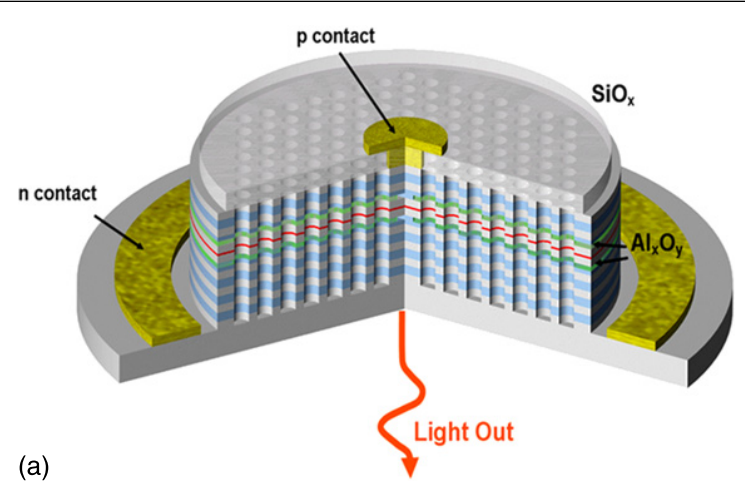

(a)

\begin{tabular}{|c|c|c|}
\hline $0.084 \mu \mathrm{m}$ & p+ & GaAs \\
\hline $0.095 \mu \mathrm{m}$ & $\mathbf{p}$ & $\mathrm{Al}_{0.80} \mathrm{Ga}_{0.20} \mathrm{As}$ \\
\hline $0.084 \mu \mathrm{m}$ & $\mathbf{p}$ & GaAs \\
\hline $0.045 \mu \mathrm{m}$ & $p$ & $\mathrm{Al}_{0.80} \mathrm{Ga}_{0.20} \mathrm{As}$ \\
\hline $0.050 \mu \mathrm{m}$ & $\mathbf{i}$ & $\mathrm{Al}_{0.98} \mathrm{Ga}_{0.02} \mathrm{As}$ \\
\hline $0.084 \mu \mathrm{m}$ & i & GaAs \\
\hline $0.084 \mu \mathrm{m}$ & $\mathbf{i}$ & GaAs \\
\hline $0.050 \mu \mathrm{m}$ & i & $\mathrm{Al}_{0.98} \mathrm{Ga}_{0.02} \mathrm{As}$ \\
\hline $0.045 \mu \mathrm{m}$ & $n$ & $\mathrm{Al}_{0.80} \mathrm{Ga}_{0.20} \mathrm{As}$ \\
\hline $0.084 \mu \mathrm{m}$ & $\mathbf{n}$ & GaAs \\
\hline $0.095 \mu \mathrm{m}$ & & $\mathrm{Al}_{0.80} \mathrm{Ga}_{0.20} \mathrm{As}$ \\
\hline $0.084 \mu \mathrm{m}$ & $\mathbf{n}$ & GaAs \\
\hline $0.334 \mu \mathrm{m}$ & $n+$ & GaAs \\
\hline \multicolumn{3}{|c|}{ GaAs S.I. Substrate } \\
\hline
\end{tabular}

Figure 1. (a) Schematic representations of a bottom emitting PC QD device with the p-metal contact placed directly above the PC microcavity. (b) Device heterostructure grown by MBE.

grown by molecular beam epitaxy (MBE). The optical gain is provided by four layers of self-assembled InGaAs QDs with a density of $10^{10} \mathrm{dots}^{-2}$ and ground state room temperature emission centred at $1.1 \mu \mathrm{m}$. The QDs are embedded in a $0.2 \mu \mathrm{m}$ thick GaAs layer surrounded by $50 \mathrm{~nm}$-thick $\mathrm{Al}_{0.98} \mathrm{Ga}_{0.02} \mathrm{As}$ layers. Two periods of p-type $\mathrm{GaAs} / \mathrm{Al}_{0.84} \mathrm{Ga}_{0.16}$ As distributed Bragg reflector (DBR) mirrors above and three periods of n-type mirrors below the active region are provided for vertical mode confinement. The QD heterostructure is shown in figure 1(b).

The device design and fabrication are described in detail in [22]. The bulk PC consists of air-holes in a triangular lattice with lattice periods $a=300 \mathrm{~nm}$ and hole diameters $\sim 190 \mathrm{~nm}$. These design parameters placed the QD photoluminescence peak $(1.1 \mu \mathrm{m})$ at the centre of the PBG for the transverse electric (TE) polarization as indicated by the plane wave expansion simulations. The electron-cyclotron resonance reactive ion etch (ECR-RIE) penetrated the heterostructure throughout the bottom DBR. The $\mathrm{Al}_{0.98} \mathrm{Ga}_{0.02} \mathrm{As}$ layers above and below the active GaAs region were wet oxidized so that only a thin post of doped dielectric material surrounded by $\mathrm{Al}_{x} \mathrm{O}_{y}$ remained under the $\mathrm{PC}$ microcavity as a path for carrier transport. The p-metal contact (dia. $\sim 1 \mu \mathrm{m}$ ) was deposited directly above the PC microcavities by e-beam lithography. A passivation layer of $\mathrm{SiO}_{2}$ was deposited and metal interconnects and contact pads were subsequently 

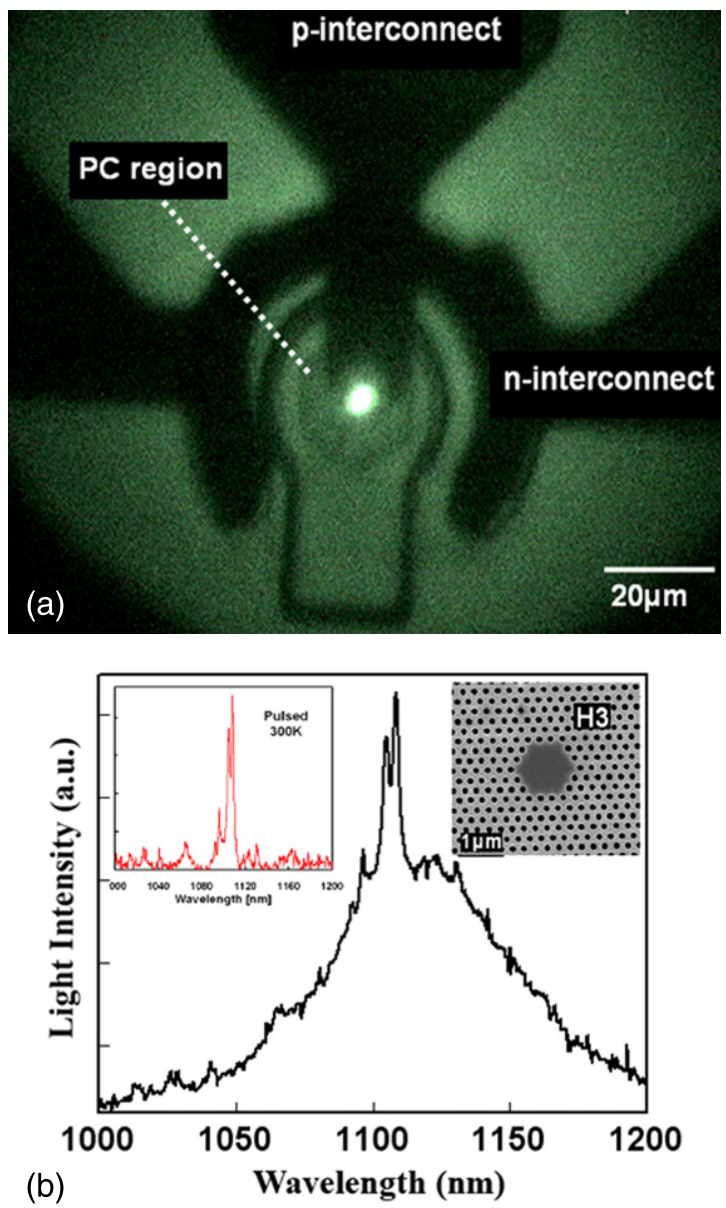

Figure 2. (a) An infrared image of the output spot of a larger cavity $\mathrm{H} 3$ showing the light being emitted from the resonant cavity region. The biased device is illuminated from the top with a white light source and the image is taken from the bottom using $100 \times$ lens focused on the polished substrate. (b) Spectral characteristics of a $\mathrm{H} 3$ device showing multiple cavity resonances supported by the PC microcavity. The spectrum after subtraction of the EL background is shown in the inset on the left. Top SEM image of the cavity is shown in the inset on the right.

lifted off. The GaAs substrate was thinned down to $150 \mu \mathrm{m}$ and polished to a mirror finish.

\subsection{Measurements and results}

The devices were characterized in pulsed mode $(10 \mu \mathrm{s}$ with $5 \%$ duty cycle) at room temperature. Due to the sub-micron size of the injection aperture (diameter $\sim 200 \mathrm{~nm}$ ) below the microcavity active region in a single-cell device, the resistance $(\Delta V / \Delta I)$ of the device was measured to be $\sim 5 \mathrm{k} \Omega$. The output light from the polished semi-insulating substrate was collimated with a $100 \times$ lens (N.A. $=1.25)$ and observed with an infrared (IR) camera. An image of the biased device with a 19 defect hexagonal microcavity (H3) illuminated from the top with a white-light source shows light emitted from the resonant cavity region surrounded by the PC region (figure 2(a)). The spectra were measured with a $0.5 \mathrm{~m}$ monochromator and germanium detector with lock-in amplification.

Emission spectra of PC microcavities with different sizes and geometries show narrow resonances corresponding to

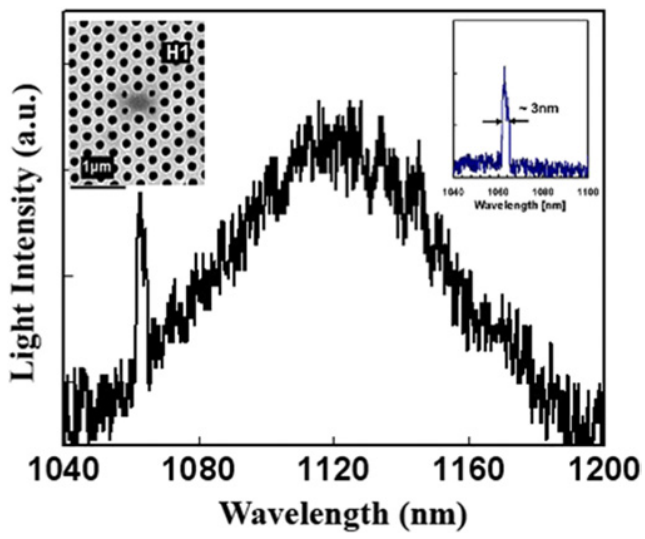

Figure 3. Emission spectra of a single-defect cavity with a split dipole degeneracy showing a $\sim 2 \mathrm{~nm}$-broad microcavity resonance at $1063 \mathrm{~nm}$; the spectrum after subtraction of the EL background is shown in the inset on the right. A top-view SEM image of the cavity is shown in the left inset.

individual microcavity defect modes. Figure $2(b)$ shows the electroluminescence (EL) spectrum observed in a hexagonal H3 microcavity (shown in the inset). The resonance spectrum after subtraction of the background EL is also shown in the inset of figure $2(b)$. Figure 3 shows the resonance spectrum of one of the fabricated single defect PC microcavities $(\mathrm{H} 1)$ formed by removing an air-hole and reducing the size $(r \sim 75 \mathrm{~nm})$ of two pairs of holes neighbouring the defect along the $\Gamma-\mathrm{X}$ direction to split the single defect dipole degeneracy. A scanning electron micrograph (SEM) of the microcavity is shown in the inset of figure 3. The resonance spectrum after background subtraction is also shown in the inset. It is estimated that there are on average 50 QDs per layer confined in the single defect microcavity. The linewidth of these resonances is determined by the microcavity $Q$ factor. In a $\mathrm{H} 1$ microcavity we observed a single $\sim 2 \mathrm{~nm}$ broad peak $(Q \sim 530)$. For a modified $\mathrm{H} 3$ cavity $\sim 1 \mathrm{~nm}$-broad multiple resonances $(Q \sim 1000)$ were observed at the QD EL peak. It should be noted that the $Q$ value of the single-defect device is considerably smaller than that observed in free-standing electrically injected lasers ( $Q \sim 2500$ ) [21], which can be attributed to low vertical $Q$ of the low-index contrast structures and non-optimized cavity designs. The devices were mounted in air between two vertical copper supports and therefore the heat sinking was not optimal.

\subsection{Discussion}

It was realized that there were several shortcomings in the fabrication of these devices. The fabrication sequence involved the chemical vapour deposition of silicon dioxide $\left(\mathrm{SiO}_{2}\right)$ as the passivation layer. The $\mathrm{SiO}_{2}$ filled the air-holes randomly during deposition, leading to possible shrinkage in the PBG. Elimination of the passivation layer was not a feasible solution since interconnect metal deposited directly on the PC infiltrated the air-holes giving rise to the possibility of contacts being shorted. At high injection currents, carriers bypassed the high resistance path provided by the post below the microcavity and instead reached the n-contacts by traversing outside the PC patterned area. As a result, IR emission could be observed from the entire mesa at high current levels. To address the fabrication discrepancies, to achieve better heat-sinking and 
also to eliminate the need for polishing the back surface of the wafer, leading to easier device handling, a surface-emitting PC microcavity light emitting device with air-bridge electrical contacts to the microcavity was conceived. This device is described next.

\section{Surface-emitting QD PC microcavity light emitter with air-bridge contacts}

\subsection{Background}

The approach to electrical injection employed by Zhou et al [19] involved the use of DBRs for out-of-plane optical confinement and PC air-holes for optical confinement in the in-plane direction. This configuration leads to lower $Q$ factors due to reduced refractive index contrast, but provides better mechanical stability and thermal conductivity. In this device, single microcavity defect mode spectra were observed. However, the aperture created by wet oxidation was larger than the PC patterned area so that optical confinement of the microcavity defect modes was provided only in the in-plane direction by 40 periods of photonic crystal. To achieve maximum $Q$ for resonant modes in a 2D PC slab structure, the optical mode must be confined in the vertical direction within a thickness $\sim 0.6$ times the lattice constant of the PC [23]. The thickness of the active region must ensure singlemode condition for guided modes in the vertical direction. The cladding thickness around the active region must be chosen appropriately to ensure that the tail of the optical mode in the vertical direction terminates as close to the active region as possible. Further, electrical charge carriers in the device described in $[20,21]$ were injected from ring contacts surrounding the PC pattern leading to carrier injection losses at the etched surfaces of the PC during device operation. These issues are addressed in the device described next.

\subsection{Design and fabrication}

The heterostructure, shown in figure 4(a) and grown by MBE, consists of five layers of self-assembled InAs QDs with ground state room temperature emission centred at $1.3 \mu \mathrm{m}$. The QDs are embedded in a GaAs layer of thickness $0.21 \mu \mathrm{m}$ and are surrounded by a $\mathrm{p}^{+}$-doped GaAs layer of thickness $0.1 \mu \mathrm{m}$ on the top and a n-doped $\mathrm{Al}_{0.98} \mathrm{Ga}_{0.02}$ As layer of thickness $0.6 \mu \mathrm{m}$ at the bottom. The devices were grown on a semiinsulating GaAs substrate with a $0.7 \mu \mathrm{m}$ thick heavily n-doped GaAs layer. The $\mathrm{Al}_{0.98} \mathrm{Ga}_{0.02} \mathrm{As}$ layer was later wet oxidized to form $\mathrm{Al}_{x} \mathrm{O}_{y}$ with refractive index 1.61. Consequently, the vertical confinement for resonant modes in the PC microcavity were provided by air on top and $\mathrm{Al}_{x} \mathrm{O}_{y}$ in the bottom except a small unoxidized post. These are referred to as oxide-clad devices. In a second device design, the oxide cladding is subsequently removed by immersing the devices in buffered hydrofluoric acid (BHF). These are referred to as air-clad devices. While resonant modes in optically excited $2 \mathrm{D}$ slab PC microcavities are confined vertically by a uniform air-cladding, electrically injected devices have an additional requirement of a high refractive index post below the centre of the microcavity to enable the transport of carriers. Three-dimensional finite difference time domain simulations were performed so that resonant modes in the PBG, with an electric field profile that
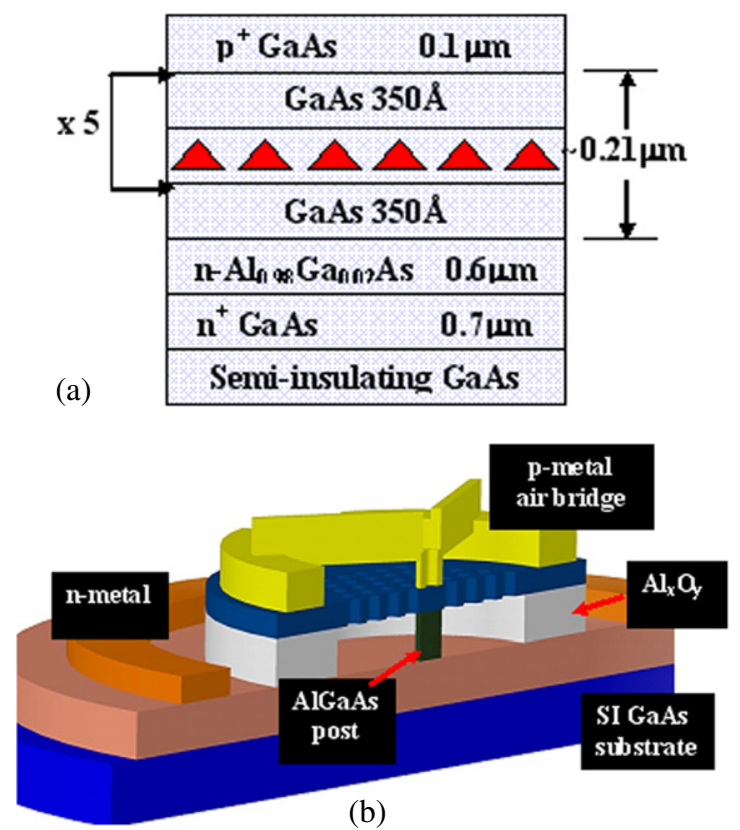

(b)

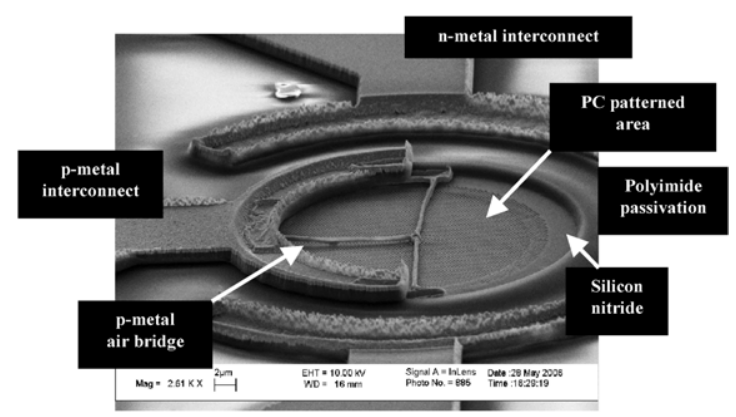

(c)

Figure 4. (a) Heterostructure grown by MBE of the top-emitting PC microcavity light-emitter with air-bridge electrical contacts (b) Schematic of the fabricated device with air-bridge p-metallic contacts to a single missing defect PC microcavity shown without the polyimide passivation and interconnect metal layers $(c)$ Top-view SEM of a finished device with air-bridge electrical contacts. Interconnect metal is deposited after polyimide passivation.

has a node at the centre of the microcavity, were located near the excited state PL peak. Modes with antinodes at the cavity centre are suppressed due to poor vertical confinement by the high index post. The position and size of the air-holes at the periphery of the cavity were adjusted [24] such that the high intensity lobes of the whispering gallery modes (WGM) are symmetrically distributed in the $\Gamma-\mathrm{K}$ and $\Gamma-\mathrm{M}$ directions, thereby improving the $Q$ of the mode.

In both oxide-clad and air-clad devices, a narrow aperture (post) of high index $\mathrm{Al}_{0.98} \mathrm{Ga}_{0.02} \mathrm{As}$ is created at the centre of the microcavity for current flow. The air-clad device is schematically shown in figure $4(b)$. The p-metal airbridges are formed in a single e-beam lithography and metal liftoff step by using bi-layer PMMA-PMAA resists that have different sensitivities to e-beam currents and by varying the ebeam exposure dose on the bridge span and posts. Standard p-metal $(\mathrm{Pd} / \mathrm{Zn} / \mathrm{Pd} / \mathrm{Au})$ with individual thicknesses of 100/200/200/4500 $\AA$, respectively, was lifted off in acetone. The air-bridges are typically $2-13 \mu \mathrm{m}$ long, $300 \mathrm{~nm}$ high and $400 \mathrm{~nm}$ wide. The width of the contacts at the surface is 
approximately $200-300 \mathrm{~nm}$. A SEM of a finished device with a single $\mathrm{H} 3$ microcavity and with air-bridge electrical contacts is shown in figure $4(c)$. As can be seen in the figure, direct electrical contact is established to the PC microcavity by a metal air-bridge $13 \mu \mathrm{m}$ long that connects to a larger p-metal pad outside the PC patterned area. It is noteworthy that the BHF etching to remove the $\mathrm{Al}_{x} \mathrm{O}_{y}$ to form air-clad devices is done after the formation of the air-bridges. These interconnects also survive post-fabrication cleaving and are therefore extremely robust.

\subsection{Measurements, results and discussion}

A typical micro-photoluminescence (micro-PL) measurement system is employed for characterizing the electroluminescence of the devices, except the exciting pump beam in micro-PL serves the purpose of a tracer beam for alignment. Wirebonded devices are mounted on a standard breadboard. The IR emission from the device is collected from the top with an infinity-corrected $100 \times$ objective lens (N.A. $=0.7$ ). A beam splitter and focusing optics direct the IR light into the input slit of a $0.75 \mathrm{~m}$ monochromator. The output from the monochromator is measured with a germanium detector using lock-in amplification.

The devices were characterized in pulsed mode (period $\sim 100 \mu \mathrm{s}$ ) at room temperature. IR emission is observed only from the centre of the microcavity. EL spectrum from an air-clad $\mathrm{H} 2$ microcavity device with a conducting post $(a=350 \mathrm{~nm})$ is shown in figure 5(a). A SEM image showing the top view of a fabricated device with an air-bridge submicron contact is shown in the inset of figure 5(a). Multiple resonances corresponding to the cavity modes are observed, whose spectral positions of the observed resonances agree well with calculated values. The resonance at $1208 \mathrm{~nm}$ has the narrowest linewidth $\sim 1.3 \mathrm{~nm}$, which translates to $Q \sim 930$. Due to good size uniformity of our $1.3 \mu \mathrm{m}$ QDs [25], with typical room temperature PL emission linewidth $\sim 30 \mathrm{meV}$, we believe that the observed resonances are microcavity defect modes and not longitudinal modes from different groups of QDs [26]. Light-current characteristics (figure 5(b)) are obtained by plotting the peak intensity of the resonant mode at $1208 \mathrm{~nm}$, after subtracting the background.

A band-structure simulation for a $\mathrm{H} 2 \mathrm{PC}$ microcavity in a free-standing slab structure shows several resonant modes. However, in electrically injected PC microcavities, where a high-index post is present below the microcavity, resonant modes with electromagnetic field energy profiles that have antinodes at the centre of the microcavity are suppressed due to poor vertical optical confinement at the location of the post. The second wet oxidation and BHF etch step ensures that only resonant modes of the whispering gallery (WG) type, with a node at the centre of the microcavity and maximum field intensity around the post, will have the necessary vertical confinement and will consequently be enhanced by the PC microcavity. Such modes have been efficiently used in electrically injected free-standing slab structures to demonstrate a single cell photonic crystal laser [21]. In the case of a $\mathrm{H} 2$ microcavity, simulations show that modes at $a / \lambda=0.29,0.28$ and 0.265 satisfy the vertical optical confinement condition. The electric field profile of
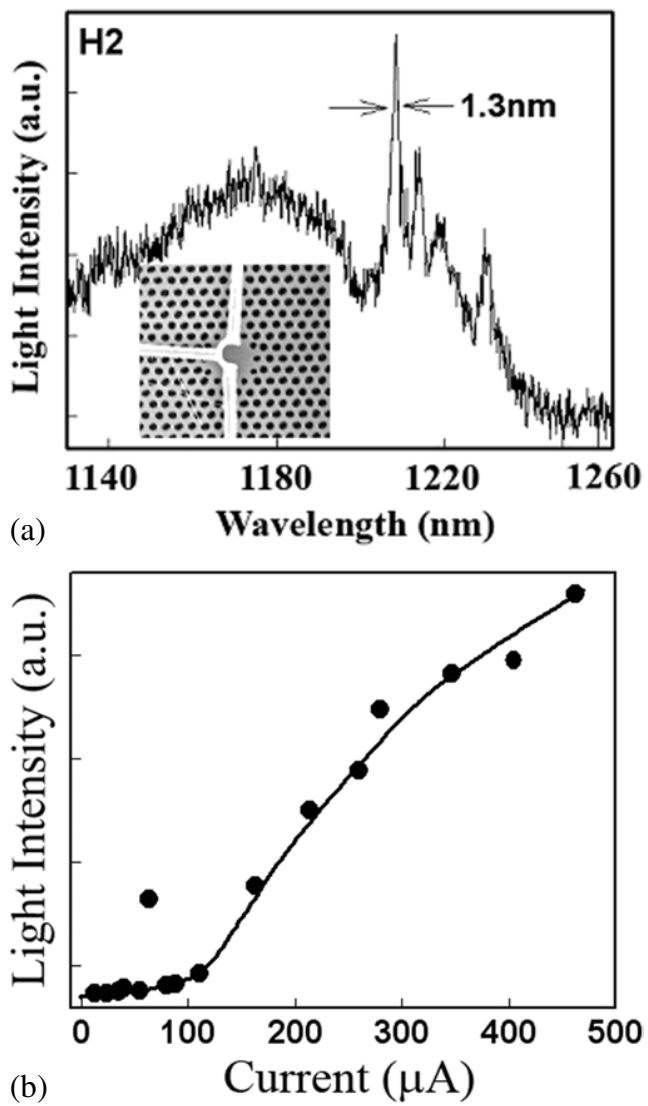

Figure 5. (a) EL spectra of a $\mathrm{H} 2$ cavity in an air-clad with central aperture post showing multiple sharp resonances. SEM of the microcavity with the air-bridge contact is shown in the inset. (b) Light-current characteristics obtained by plotting the peak intensity of the resonant mode at $1208 \mathrm{~nm}$.

these resonant modes is shown in figure 6. The hexapole mode at $a / \lambda=0.29$ corresponds to the mode observed at $1208 \mathrm{~nm}$. The maxima of the electric field intensity of the hexapole modes at $a / \lambda=0.28$ and 0.29 are in close proximity to the high refractive index post at the centre of the microcavity. Due to a small uncertainty in the diameter of the conducting post, which depends on the etch rate of $\mathrm{Al}_{x} \mathrm{O}_{y}$, a small fraction of the electric field intensity of the hexapole modes can overlap with the high refractive index post, thereby lowering the $Q$ of the mode. Since the electric field energy density of the WGM at $a / \lambda=0.265$ is concentrated farther away from the conducting post at the centre of the microcavity, we expect that the linewidth of the WGM will be narrower than the linewidth of the mode shown in figure $5(a)$. In the design of the PC microcavity, the air-holes at the periphery of the microcavity were shifted outwards and reduced in size to improve the $Q$ of the WGM [24]. Due to fabrication tolerances and large sensitivity of the frequency of the WGM on the size of the nearest neighbour air-holes, we believe the WGM in our device is shifted to longer wavelengths where the emission intensity of QDs is low. The presence of the background PL emission in optically excited PC microcavities [2] can be explained by the fact that active material in the vicinity of the microcavity is excited due to the large excitation spot size ( $\sim 2 \mu$ m diameter $)$ of the pump beam. However, when the $Q$ of the resonant mode is exceptionally high, the background PL intensity is 


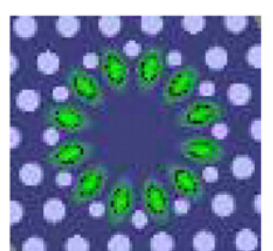

a/ $\lambda=0.265$

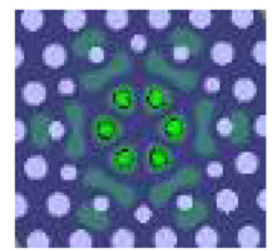

$\mathrm{a} / \lambda=\mathbf{0 . 2 8}$

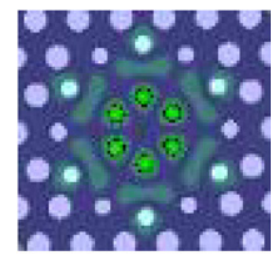

$\mathrm{a} / \lambda=0.29$
Figure 6. TE field profiles of resonant modes in a $\mathrm{H} 2$ microcavity with nodes at the centre of the microcavity. Mode at $a / \lambda=0.265$ is the whispering gallery mode. Air-holes at the periphery of the microcavity are shifted outward and modified in size as described in [24].

negligible compared with the intensity of the high- $Q$ resonant mode [9]. By tuning the lattice constant of the PC, we expect to relocate the high-Q WGM to the peak of the EL spectrum and significantly suppress the background EL intensity. Under these circumstances, the background EL emission intensity will be significantly smaller than the peak intensity of the resonant WGM and the device is expected to display true 'lasing' behavior. The chances of achieving coherent emission can be increased by forming an active region with QDs having higher gain and shallower confinement than the $1.3 \mu \mathrm{m}$ QDs [27] employed in this study.

The signature of all the resonant modes supported by the PC microcavity can be observed in the EL spectra from very low current injection levels. The linewidths of the resonant modes is independent of the carrier injection level. The series resistance of the $\mathrm{H} 2$ microcavity devices was measured to be $2.8 \mathrm{k} \Omega$. Compared with GaAs that has an electron mobility $\sim 8500 \mathrm{~cm}^{2} \mathrm{~V} \mathrm{~s}^{-1}$, the mobility is much smaller in $\mathrm{Al}_{0.98} \mathrm{Ga}_{0.02} \mathrm{As}\left(100 \mathrm{~cm}^{2} \mathrm{~V}-\mathrm{s}^{-1}\right)$. Further, the narrow effective aperture for carrier injection created by wet oxidation/wet etching increases the series resistance of the device. The high $\mathrm{Al}$ concentration was chosen to achieve the maximum refractive index contrast for optical modes at the centre of the microcavity and also to ensure a sufficiently high wet oxidation rate to form $\mathrm{Al}_{x} \mathrm{O}_{y}$. The series resistance is calculated as several $\mathrm{k} \Omega$ in a single cell device. Spectral measurements of single cell devices are currently in progress.

To gain a better understanding of the role and significance of vertical optical confinement in these devices, EL spectra were also measured, prior to wet etching with $\mathrm{BHF}$, for a pedestal-mounted $\mathrm{H} 2$ microcavity with bottom $\mathrm{Al}_{x} \mathrm{O}_{y}$ cladding (oxide-clad device). Only one broad resonance (linewidth $\sim 3 \mathrm{~nm}(Q \sim 400)$ ) is observed (figure 7) at $1160 \mathrm{~nm}$, in sharp contrast to the narrow resonances observed for air-clad devices. It is therefore clear that strong optical confinement with high refractive index contrast in the vertical direction is necessary in 2D PC slab pedestal mounted microcavities to observe high- $Q$ resonances. Similar results were obtained with $\mathrm{H} 3$ and other higher-dimensional hexagonal microcavities in the triangular lattice PC devices. Heat dissipation from the active region can occur only through the doped dielectric pedestal below the microcavity in air-clad devices, in contrast to oxide-clad devices where the oxide aids in dissipating heat.

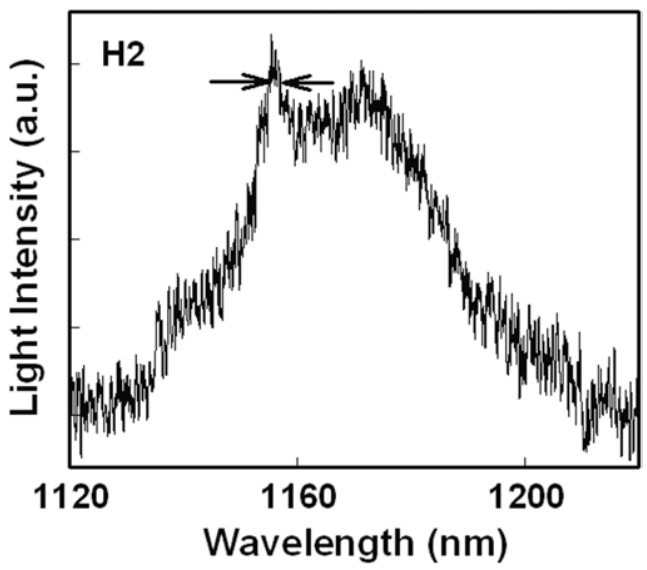

Figure 7. EL spectra of a $\mathrm{H} 2$ cavity in an oxide-clad structure (SEM of the microcavity in the inset) with central aperture post showing a single $3.5 \mathrm{~nm}$ broad resonance.

\section{Surface-emitting QD PC microcavity light-emitting arrays}

An optically pumped PC nanocavity laser array was recently demonstrated with higher output power than an isolated nanocavity laser [28]. The metallic air-bridges fabricated for single microcavity devices can also be employed to make electrical contacts to multiple cavities in parallel and will enable the realization of dense arrays of PC light sources. Using the fabrication procedure described earlier, we have developed a robust technique for fabricating such arrays. As shown in the SEM images in figures $8(a)$ and $(b)$, respectively, bridge contacts are made to $37 \mathrm{H} 5$ oxide-clad cavities from the same outer p-metal pad. Figure $8(c)$ shows IR emission from a $\mathrm{H} 5$ microcavity array. The 37 microcavities are confined in a circular area of diameter $45 \mu \mathrm{m}$ and are separated by $4-5$ periods of $P C$ air-holes. The number of peaks observed in the spectral output and their wavelengths were the same for the array as for a single microcavity; however, the signal-to-noise ratio was much larger for the array. Similar arrays were realized with $19 \mathrm{H} 7$ microcavities and $108 \mathrm{H} 2$ cells, shown in figures $9(a)$ and $9(b)$, respectively, with the light emission shown in the insets. Both arrays were also made of oxide clad devices. In the 108 element array, the air-bridges connecting adjacent devices are only $2 \mu \mathrm{m}$ in length. To the best of our knowledge, this is the first demonstration of such densely integrated electrically injected QD PC microcavity light-emitting arrays.

It is observed in the IR emission images that the intensity from individual microcavities in the array are different. We believe that the varying intensity is due to variations in the size of the current aperture created below the microcavity by the second wet oxidation step. The variation in resistance between individual microcavities is much larger for $\mathrm{H} 2$ cavities $(\sim 2.8 \mathrm{k} \Omega)$ than for $\mathrm{H} 5$ or $\mathrm{H} 7$ cavities $(\sim 60-100 \Omega)$. As a result, the number of bright microcavities in the $\mathrm{H} 2$ array is less than in the H5 or H7 PC arrays. By better characterization of the wet oxidation and etching steps, this variation can be minimized, to achieve uniform light emission. 


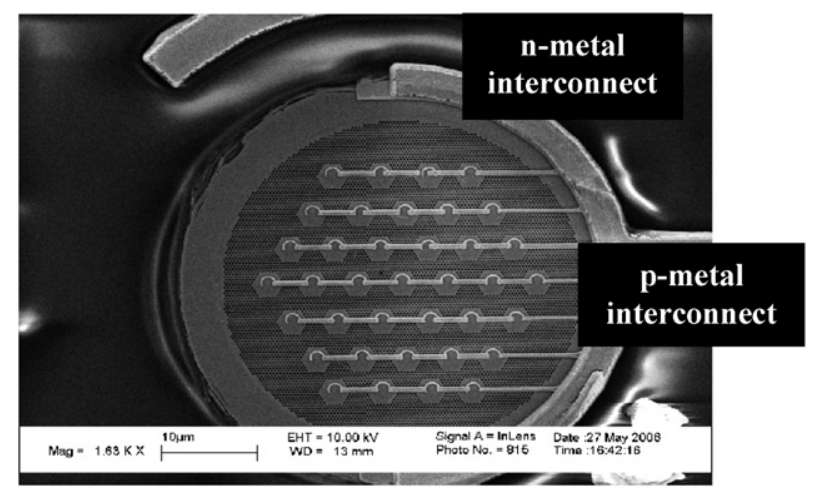

(a)

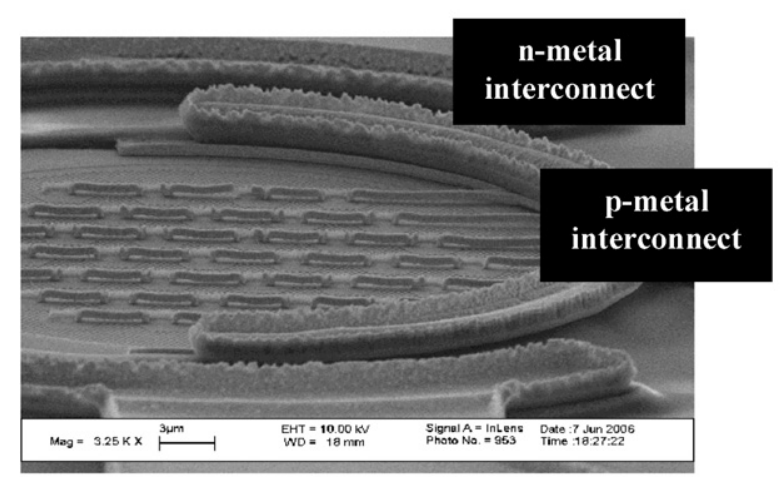

(b)

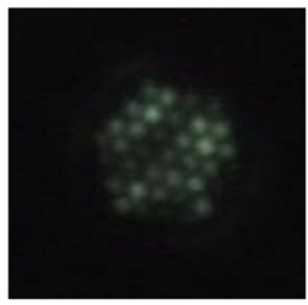

(c)

Figure 8. SEM images showing (a) top view and $(b)$ side view of an array of $37 \mathrm{H} 5$ microcavities joined by air-bridge electrical contacts. (c) Infrared (IR) emission observed through an IR viewer, from the array of $\mathrm{H} 2$ microcavities. The relative brightness of the microcavities is determined by the resistance of the individual microcavities, due to fabrication tolerances that determine the size of the current aperture.

\section{Conclusion}

We have described the characteristics of electrically injected PC microcavity light sources and arrays of such devices. Schemes of electrical carrier injection to bottom-emitting and top-emitting devices, with a narrow current aperture defined by wet oxidation of AlGaAs, have been investigated. It is seen that in surface emitting devices and arrays, metallic air-bridge contacts to the microcavity enables direct carrier injection and eliminates problems of injected carrier recombination and loss present in devices where a top ring contact is formed outside the photonic crystal. The modes of the microcavity are identified in the output spectra. The linewidths of the resonant modes observed in the output spectra are dependent on the cavity quality factor, which in turn depends on the nature of the cladding of the active region. As expected,

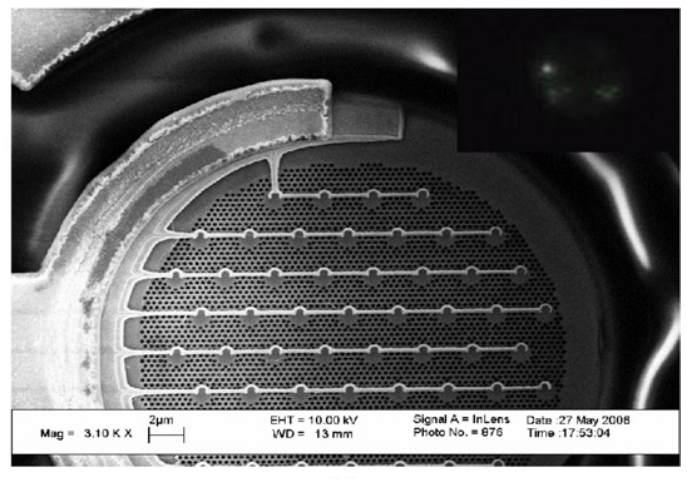

(a)

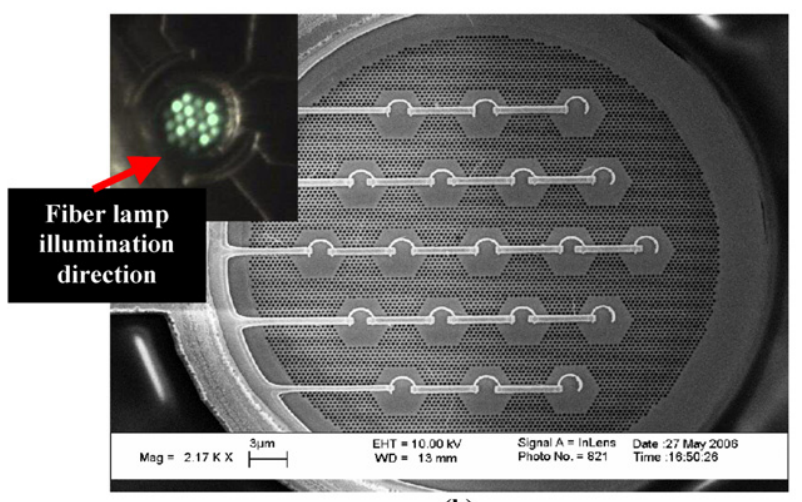

(b)

Figure 9. (a) SEM image showing top view of an array of $108 \mathrm{H} 2$ microcavities connected by air-bridge nano contacts. Infrared (IR) emission observed through an IR viewer is shown in the inset. (b) SEM image showing top view of an array of $19 \mathrm{H} 7$ microcavities interconnected by air-bridge contacts. Inset shows the emission observed from the array.

the oxide-clad devices display larger linewidths $(2-3 \mathrm{~nm})$ than the air-clad membrane-like devices $(1.3 \mathrm{~nm})$. While some coherence is observed in the output spectra, an order of magnitude improvement in the linewidth will lead to lasing. The air-bridge technology was also successfully employed to realize dense arrays of electrically injected PC microcavity light sources.

\section{Acknowledgments}

This work was performed at the Michigan Nanofabrication Facility (MNF) and has been supported by the Center for Optoelectronic Nanostructures Semiconductor Technologies, a DARPA UPR award, under Contract \#R0011-04-1-0040 and the NSF under Grant BES-0412387.

\section{References}

[1] Englund D, Fattal D, Waks E, Solomon G, Zhang B, Nakaoka T, Arakawa Y, Yamamoto Y and Vuckovic J 2005 Controlling the spontaneous emission rate of single quantum dots in a two-dimensional photonic crystal Phys. Rev. Lett. 95013904

[2] Painter O, Lee R K, Scherer A, Yariv A, O’Brien J D, Dapkus P D and Kim I 1999 Two-dimensional photonic band gap defect mode laser Science 284 1819-21

[3] Song B S, Asano T, Akahane Y, Tanaka Y and Noda S 2005 Multichannel add/drop filter based on in-plane hetero-photonic crystals J. Lightwave Technol. 23 1449-55 
[4] Smith C J M, Benisty H, Olivier S, Rattier M, Weisbuch C, Krauss T F, De La Rue R M, Houdre R and Oesterle U 2000 Low-loss channel waveguides with two-dimensional photonic crystal boundaries Appl. Phys. Lett. 77 2813-5

[5] Lončar M, Scherer A and Qiu Y M 2003 Photonic crystal laser sources for chemical detection Appl. Phys. Lett. 82 4648-50

[6] Chakravarty S, Topol'anćik J, Bhattacharya P and Chakrabarti S 2005 Ion-detection with photonic crystal microcavities Opt. Lett. 30 2578-80

[7] Sharkawy A, Shi S and Prather D W 2003 Implementation of optical vias in high-density photonic crystal optical networks J. Microlithogr. Microfab. Microsyst. 2 300-8

[8] Vuckovic J and Yamamoto Y 2003 Photonic crystal microcavities for cavity quantum electrodynamics with a single quantum dot Appl. Phys. Lett. 82 2374-6

[9] Yoshie T, Scherer A, Hendrickson J, Khitrova G, Gibbs H M, Rupper G, Ell C, Shchekin O B and Deppe D G 2004 Vacuum Rabi splitting with a single quantum dot in a photonic crystal nanocavity Nature 432 200-3

[10] Yablonovitch E 1987 Inhibited spontaneous emission in solid-state physics and electronics Phys. Rev. Lett. 58 2059-63

[11] John S 1987 Strong localization of photons in certain disordered dielectric superlattices Phys. Rev. Lett. 58 2486-9

[12] Sabarinathan J, Bhattacharya P, Zhu D H, Kochman B, Zhou W D and Yu P C 2001 Submicron three-dimensional infrared $\mathrm{GaAs} / \mathrm{Al}_{x} \mathrm{O}_{y}$-based photonic crystal using single-step epitaxial growth Appl. Phys. Lett. 78 3024-6

[13] Imada M, Lee L H, Okano M, Kawashima S and Noda S 2006 Development of three-dimensional photonic-crystal waveguides at optical communication wavelengths Appl. Phys. Lett. 88171107

[14] Ralph T C, Gilchrist A, Milburn G J, Munro W J and Glancy S 2003 Quantum computation with optical coherent states Phys. Rev. A 68 042319/1-042319/11

[15] Gisin N, Ribordy G, Tittel W and Zbinden H 2000 Quantum cryptography Rev. Mod. Phys. 14 145-95

[16] Bouwmeester D, Pan J W, Mattle K, Eible M, Weinfurter H and Zeilinger A 1997 Experimental quantum teleportation Nature 390 575-8
[17] Yang T, Lipson S, O’Brien J D and Deppe D G 2005 InAs quantum dot photonic crystal lasers and their temperature dependence IEEE Photon. Technol. Lett. 17 2244-6

[18] Yoshie T, Shchekin O B, Chen H, Deppe D G and Scherer A 2002 Quantum dot photonic crystal lasers Electron. Lett. 38 967-8

[19] Zhou W D, Sabarinathan J, Bhattacharya P, Kochman B, Berg E W, Yu P C and Pang S 2001 Characteristics of a photonic band gap single defect microcavity electroluminescent device IEEE J. Quantum Electron. 37 1153-60

[20] Sabarinathan J, Bhattacharya P, Krishna S, Yu P C, Cheng J and Steel D J 2002 An electrically injected InAs/GaAs quantum-dot photonic crystal microcavity light-emitting diode Appl. Phys. Lett. 81 3876-8

[21] Park H G, Kim S H, Kwon S H, Ju Y G, Yang J K, Baek J H, Kim S B and Lee Y H 2004 Electrically driven single cell photonic crystal laser Science 305 1444-8

[22] Topol'ančik J, Chakravarty S, Bhattacharya P and Chakrabarti S 2006 Electrically injected quantum dot photonic crystal microcavity light sources $O p t$. Lett. 31 232-4

[23] Painter O, Vuckovic J and Scherer A 1999 Defect modes of a two-dimensional photonic crystal in an optically thin dielectric slab J. Opt. Soc. Am. B-Opt. Phys. 16 275-85

[24] Ryu H Y, Notomi M, Kim G H and Lee Y H 2004 High quality factor whispering gallery mode in the photonic crystal hexagonal disk cavity Opt. Express 121708

[25] Mi Z and Bhattacharya P 2005 Molecular-beam epitaxial growth and characteristics of highly uniform InAs/GaAs quantum dot layers J. Appl. Phys. 98023510

[26] Harris L, Mowbray D J, Skolnick M S, Hopkinson M and Hill G 1998 Emission spectra and mode structure of InAs/GaAs self-organized quantum dot lasers Appl. Phys. Lett. 73969

[27] Strauf S, Hennessy K, Rakher M T, Choi Y-S, Badolato A, Andreani L C, Hu E L, Petroff P M and Bouwmeester D 2006 Self-tuned quantum dot gain in photonic crystal lasers Phys. Rev. Lett. 96127404

[28] Altug H and Vuckovic J 2005 Photonic crystal nanocavity array laser Opt. Express 13 8819-28 